\title{
Flat-branched semisimplicial complexes: a versatile tool for aperiodic solids
}

\author{
P. Kalugin ${ }^{1}$, A. Katz ${ }^{2}$ \\ ${ }^{1}$ Laboratoire de Physique des Solides, CNRS, Universit'e Paris-Sud, Universit'e Paris-Saclay, F-91405 Orsay, France, \\ ${ }^{2}$ Directeur de recherche honoraire, CNRS, France
}

kalugin@lps.u-psud.fr

We introduce flat-branched semisimplicial (FBS) complexes as a universal language to describe aperiodic structures of finite local complexity. An FBS-complex naturally represents the set of local atomic arrangements occurring in the structure. It includes both metric and combinatorial data; the flexibility of the latter allows for incorporation of structural constraints on a longer range. An FBScomplex can embody "local rules" of any kind, whether or not they impose a perfect long-range order. We propose an algorithm for exploration of local rules in terms of an FBS-complex directly from the phased diffraction data [1]. The FBS complex describing a structure entirely determines the density of atomic species, and yields experimentally verifiable constraints on their contribution to the structure factors [2].

[1] Kalugin, P, Katz, A. (2019). Acta Cryst. A 75(5), 669-693.

[2] Kalugin, P, Katz, A. (2021) in preparation

Keywords: aperiodic solids, quasicrystals, matching rules, diffraction, density 